\title{
NORMALITY CRITERIA FOR FAMILIES OF MEROMORPHIC FUNCTIONS
}

\author{
SANJAY KUMAR AND POONAM RANI
}

\begin{abstract}
In this paper we prove some normality criteria for a family of meromorphic functions, which involves the zeros of certain differential polynomials generated by the members of the family.
\end{abstract}

\section{INTRODUCTION AND MAIN RESULTS}

An important aspect of the theory of complex analytic functions is to find normality criteria for families of meromorphic functions. The notion of normal families was introduced by Paul Montel in 1907. Let us begin by recalling the definition. A family of meromorphic (holomorphic) functions defined on a domain $D \subset \mathbb{C}$ is said to be normal in the domain, if every sequence in the family has a subsequence which converges spherically uniformly on compact subsets of $D$ to a meromorphic (holomorphic) function or to $\infty$.

According to Bloch's principle, a family of meromorphic functions in a domain $D$ possessing a property which reduces a meromorphic function in the plane to a constant, makes the family normal in the domain $D$. Although Bloch's principle is not true in general, many authors established normality criteria for families of meromorphic functions having such properties.

Hayman [8] proved a result which states that: Let $n \geq 5$ be a positive integer and $a(\neq 0), b$ be two finite complex numbers. If a meromorphic function $f$ in $\mathbb{C}$ satisfies, $f^{\prime}+a f^{n} \neq b$, then $f$ is a constant. The normality related to this result was conjectured by Hayman. Confirming this conjecture of Hayman, Drasin [5] proved a normality criterion for holomorphic function which says: Let $\mathcal{F}$ be a family of holomorphic functions in the unit disc $\Delta$, and for a fixed $n \geq 3$ and $a(\neq 0), b$ are finite complex numbers suppose that for each $f \in \mathcal{F}, f^{\prime}+a f^{n} \neq b$ in $\Delta$. Then $\mathcal{F}$ is normal. Schwick [13] also proved a normality criterion which confirms the following result: Let $k, n$ be positive integers with $n \geq k+4$ and $a, b \in \mathbb{C}$ with $a \neq 0$. Let $\mathcal{F}$ be a family of meromorphic functions in $a$ domain D. If for each $f \in \mathcal{F}, f^{(k)}-a f^{n} \neq b$, then $\mathcal{F}$ is normal.

Similar to above results Fang and Zalcman [7] proved a normality criterion which states: Let $\mathcal{F}$ be a family of meromorphic functions in a domain $D$, let $n \geq 2$ be a positive integer, and let $a(\neq 0), b \in \mathbb{C}$. If for each $f \in \mathcal{F}$, all zeros of $f$ are multiple and $f+a\left(f^{\prime}\right)^{n} \neq b$

2010 Mathematics Subject Classification. 30D45, 30D35.

Key words and phrases. meromorphic functions, holomorphic functions, normal families, Zalcman's lemma.

The research work of the second author is supported by research fellowship from CSIR India. 
on $D$, then $\mathcal{F}$ is normal on $D$. Yan $\mathrm{Xu}$ et al [16] extended this result and proved: Let $\mathcal{F}$ be a family of meromorphic functions in a domain $D$, let $k, n(\geq k+1)$ be two positive integers, and let $a(\neq 0), b \in \mathbb{C}$. If for each $f \in \mathcal{F}$, all zeros of $f$ are of multiplicity at least $k+1$ and $f+a\left(f^{(k)}\right)^{n} \neq b$ on $D$, then $\mathcal{F}$ is normal on $D$. They conjectured that the conclusion of their result still holds for $n \geq 2$. Confirming their conjecture, C. L. Lei et al [11] proved Let $\mathcal{F}$ be a family of meromorphic functions in a domain $D$, let $n \geq 2$ be a positive integer, and let $a(\neq 0), b \in \mathbb{C}$. If for each $f \in \mathcal{F}$, all zeros of $f$ are of multiplicity at least $k+1$ and $f+a\left(f^{k}\right)^{n} \neq b$ on $D$, then $\mathcal{F}$ is normal on $D$.

On the other side, Xia and $\mathrm{Xu}$ [14] proved the following result:

Theorem A. Let $\mathcal{F}$ be a family of meromorphic functions defined on a domain $D \subset \mathbb{C}$. Let $\psi(\not \equiv 0)$ be a holomorphic function in $D$, and $k$ be a positive integer. Suppose that for every function $f \in \mathcal{F}, f \neq 0, f^{(k)} \neq 0$ and all zeros of $f^{(k)}-\psi(z)$ have multiplicities at least $\frac{k+2}{k}$. If for $k=1, \psi$ has only zeros with multiplicities at most 2 , and for $k \geq 2, \psi$ has only simple zeros, then $F$ is normal in $D$.

Recently, B. Deng et al [3] studied the case where $f^{(k)}-a f^{n}-b$ has some distinct zeros in $D$ and they proved the following results:

Theorem B. Let $\mathcal{F}$ be a family of meromorphic functions in a domain $D$, let $m, n, k$ be three positive integers such that $n \geq k+m+2$, and let $a(\neq 0), b \in \mathbb{C}$. If for each $f \in \mathcal{F}$, all zeros of $f$ are of multiplicity at least $k$ and $f^{(k)}-a f^{n}-b$ has at most $m$ distinct zeros, then $\mathcal{F}$ is normal on $D$.

It is natural to ask if $f^{(k)}-a f^{n}-\psi$ has zeros with some multiplicities for a holomorphic function $\psi(z) \neq 0$. In this paper we investigate this situation and prove the following results:

Theorem 1.1. Let $\mathcal{F}$ be a family of meromorphic functions in a domain $D$. Let $d, k$ and $m$ be positive integers such that $\frac{k+1}{d}+\frac{k+2}{2 m}<\frac{1}{2}$. Let $a(z)$ and $\psi(z)(\neq 0)$ be holomorphic functions in D. If for each $f \in \mathcal{F}$, all zeros of $f$ are of multiplicity at least $d$, poles of $f$ are multiple and for a positive integer $n$, all zeros of $f^{(k)}(z)-a(z) f^{n}(z)-\psi(z)$ are of multiplicity at least $m$ in $D$, then $\mathcal{F}$ is normal in $D$.

For a family of holomorphic functions we have the following strengthened version:

Theorem 1.2. Let $\mathcal{F}$ be a family of holomorphic functions in a domain $D$. Let $d, k$, and $m$ be positive integers such that $\frac{k+1}{d}+\frac{1}{m}<1$. Let $a(z)$ and $\psi(z)(\neq 0)$ be holomorphic functions in $D$. If for each $f \in \mathcal{F}$, all zeros of $f$ are of multiplicity at least $d$ and for a positive integer $n$, all zeros of $f^{(k)}(z)-a(z) f^{n}(z)-\psi(z)$ are of multiplicity at least $m$ in $D$, then $\mathcal{F}$ is normal in $D$.

Corollary 1.3. Let $\mathcal{F}$ be a family of holomorphic functions on a domain $D$, let $k, m, n$ be three positive integers. If for each $f \in \mathcal{F}, f$ has zeros of multiplicities at least $m$, all zeros of $f^{(k)}-1$ are of multiplicity at least $n$ in $D$ and $\frac{k+1}{m}+\frac{1}{n}<1$, then $\mathcal{F}$ is normal in D.

The following example shows that $n$ can't be zero in Theorem 1.2 . 
Example 1.4. Let $D:=\{z \in \mathbb{C}:|z|<1\}$. Consider the family $\mathcal{F}:=\left\{j z^{5}: j \in \mathbb{N}\right\}$ on $D$. Let $a(z)=e^{z}, \psi(z)=-e^{z}$. Then for $n=0$ and $k=1, f^{(k)}(z)-a(z) f^{n}(z)-\psi(z)$ has zeros of multiplicity 4. Clearly, all conditions of Theorem 1.2 are satisfied but $\mathcal{F}$ is not normal in $D$.

The next example shows that the condition $\psi(z) \neq 0$ can not be relaxed.

Example 1.5. Let $D:=\{z \in \mathbb{C}:|z|<1\}$. Consider the family $\mathcal{F}:=\left\{j z^{4}: j \in \mathbb{N}\right\}$ on $D$. Let $a(z)=0, \psi(z)=z^{d-1}$. Then for $k=1, f^{(k)}(z)-a(z) f^{n}(z)-\psi(z)$ has zeros of multiplicity 3. Clearly, all conditions of Theorem 1.2 are satisfied but $\mathcal{F}$ is not normal in $D$.

The following example supports our Theorem 1.2 .

Example 1.6. Let $D:=\{z \in \mathbb{C}: z \neq 0\}$. Consider the family $\mathcal{F}:=\left\{j z^{4}: j \in \mathbb{N}\right\}$ on $D$. Let $a(z)=-z^{-4}, \psi(z)=-z^{2} / 4$. Then for $n=2$ and $k=1, f^{(k)}(z)-a(z) f^{n}(z)-\psi(z)$ has zeros of multiplicity 2 in $D$ and all conditions of Theorem 1.2 are satisfied. $\mathcal{F}$ is normal in $D$.

We also investigate this situation for $f(z)+a(z)\left(f^{(k)}\right)^{n}(z)-\psi(z)$ and prove the following result:

Theorem 1.7. Let $\mathcal{F}$ be a family of meromorphic functions in a domain $D$. Let $d, k, m$ and $n(\geq 2)$ be four positive integers such that $\frac{n k+1}{(n-1) d}+\frac{n(k+1)}{m(n-1)}<1$. Let $a(z)(\not \equiv 0)$ and $\psi(z)(\neq 0)$ be holomorphic functions in $D$. If for each $f \in \mathcal{F}$, all zeros of $f$ are of multiplicity at least $d$ and all zeros of $f(z)+a(z)\left(f^{(k)}\right)^{n}(z)-\psi(z)$ are of multiplicity at least $m$, then $\mathcal{F}$ is normal in $D$.

For a family of holomorphic functions we have the following strengthened version:

Theorem 1.8. Let $\mathcal{F}$ be a family of holomorphic functions in a domain $D$. Let $d, k, m$ and $n(\geq 2)$ be four positive integers such that $\frac{n k+1}{n d}+\frac{1}{m}<1$. Let $a(z)(\not \equiv 0)$ and $\psi(z)(\neq 0)$ be holomorphic functions in D. If for each $f \in \mathcal{F}$, all zeros of $f$ are of multiplicity at least $d$ and all zeros of $f(z)+a(z)\left(f^{(k)}\right)^{n}(z)-\psi(z)$ are of multiplicity at least $m$, then $\mathcal{F}$ is normal in $D$.

The following example supports Theorem 1.8

Example 1.9. Let $D:=\{z \in \mathbb{C}:|z|>0\}$. Consider the family $\mathcal{F}:=\left\{j z^{4}: j \in \mathbb{N}\right\}$ on $D$. Let $a(z)=z^{-1} / 16, \psi(z)=-z^{3} / 4$. Then for $k=1$ and $n=2, f(z)+a(z)\left(f^{(k)}\right)^{n}(z)-\psi(z)$ has zeros of multiplicity 2 and all conditions of Theorem 1.8 are satisfied. Clearly, $\mathcal{F}$ is normal in $D$.

\section{Some Notation and Results of Nevanlinna theory}

Let $\Delta=\{z:|z|<1\}$ be the unit disk. We use the following standard functions of value distribution theory, namely

$$
T(r, f), m(r, f), N(r, f) \text { and } \bar{N}(r, f) .
$$

We let $S(r, f)$ be any function satisfying

$$
S(r, f)=o(T(r, f)), \text { as } r \rightarrow+\infty,
$$


possibly outside a set of finite measure.

First Fundamental Theorem. Let $f$ be a meromorphic function on $\mathbb{C}$ and $a$ be $a$ complex number. Then

$$
T\left(r, \frac{1}{f-a}\right)=T(r, f)+O(1) .
$$

Logarithmic Derivative Lemma. Let $f$ be a non-constant meromorphic function on $\mathbb{C}$, and let $k$ be a positive integer. Then the equality

$$
m\left(r, \frac{f^{(k)}}{f}\right)=S(r, f)
$$

holds for all $r \in[1, \infty)$ excluding a set of finite measure.

\section{Preliminary Results}

In order to prove our results we need the following Lemmas. The well known Zalcman Lemma is a very important tool in the study of normal families. The following is a new version due to Zalcman [20].

Lemma 3.1. 20, 19] Let $\mathcal{F}$ be a family of meromorphic functions in the unit disk $\Delta$, with the property that for every function $f \in \mathcal{F}$, the zeros of $f$ are of multiplicity at least $l$ and the poles of $f$ are of multiplicity at least $k$. If $\mathcal{F}$ is not normal at $z_{0}$ in $\Delta$, then for $-l<\alpha<k$, there exist

(1) a sequence of complex numbers $z_{n} \rightarrow z_{0},\left|z_{n}\right|<r<1$,

(2) a sequence of functions $f_{n} \in \mathcal{F}$,

(3) a sequence of positive numbers $\rho_{n} \rightarrow 0$,

such that $g_{n}(\zeta)=\rho_{n}^{\alpha} f_{n}\left(z_{n}+\rho_{n} \zeta\right)$ converges to a non-constant meromorphic function $g$ on $\mathbb{C}$ with $g^{\#}(\zeta) \leq g^{\#}(0)=1$. Moreover, $g$ is of order at most two. Here, $g^{\#}(z)=\frac{\left|g^{\prime}(z)\right|}{1+|g(z)|^{2}}$ is the spherical derivative of $g$.

Remark 3.2. In Lemma 3.1, if $\mathcal{F}$ is a family of holomorphic functions, then by Hurwitz's Theorem the limit function $g$ is a non-constant entire function and the order of $g$ is at most 1.

The following lemma is Milloux's inequality.

Lemma 3.3. [9, 17, 18] Suppose $f(z)$ is a non-constant meromorphic function in the complex plane and $k$ is a positive integer. Then

$$
T(r, f)<\bar{N}(r, f)+N\left(r, \frac{1}{f}\right)+\bar{N}\left(r, \frac{1}{f^{(k)}-1}\right)-N\left(r, \frac{1}{f^{(k+1)}}\right)+S(r, f) .
$$

Let $f$ be a non-constant meromorphic function in $\mathbb{C}$. A differential polynomial $P$ of $f$ is defined by $P(z):=\sum_{i=1}^{n} \alpha_{i}(z) \prod_{j=0}^{p}\left(f^{(j)}(z)\right)^{S_{i j}}$, where $S_{i j}$ 's are non-negative integers and 
$\alpha_{i}(z) \not \equiv 0$ are small functions of $f$, that is $T\left(r, \alpha_{i}\right)=o(T(r, f))$. The lower degree of the differential polynomial $P$ is defined by

$$
d(P):=\min _{1 \leq i \leq n} \sum_{j=0}^{p} S_{i j}
$$

The following result was proved by Dethloff et al. in [4].

Lemma 3.4. Let $a_{1}, \ldots, a_{q}$ be distinct non-zero complex numbers. Let $f$ be a nonconstant meromorphic function and let $P$ be a non-constant differential polynomial of $f$ with $d(P) \geq 2$. Then

$$
T(r, f) \leq\left(\frac{q \theta(P)+1}{q d(P)-1}\right) \bar{N}\left(r, \frac{1}{f}\right)+\frac{1}{q d(P)-1} \sum_{j=1}^{q} \bar{N}\left(r, \frac{1}{P-a_{j}}\right)+S(r, f),
$$

for all $r \in[1,+\infty)$ excluding a set of finite Lebesgue measure, where $\theta(P):=\max _{1 \leq i \leq n} \sum_{j=0}^{p} j S_{i j}$.

Moreover, in the case of an entire function, we have

$$
T(r, f) \leq\left(\frac{q \theta(P)+1}{q d(P)}\right) \bar{N}\left(r, \frac{1}{f}\right)+\frac{1}{q d(P)} \sum_{j=1}^{q} \bar{N}\left(r, \frac{1}{P-a_{j}}\right)+S(r, f),
$$

for all $r \in[1,+\infty)$ excluding a set of finite Lebesgue measure.

This result was proved by Hinchliffe in [10] for $q=1$.

\section{Proof of Main Results}

Proof of Theorem 1.1. Since normality is a local property, we assume that $D=\Delta$. Suppose that $\mathcal{F}$ is not normal in $\Delta$. Then there exists at least one point $z_{0}$ such that $\mathcal{F}$ is not normal at the point $z_{0}$ in $\Delta$. Without loss of generality we assume that $z_{0}=0$. Then by Lemma 3.1, for $\alpha=-k$ there exist

(1) a sequence of complex numbers $z_{j} \rightarrow 0,\left|z_{j}\right|<r<1$,

(2) a sequence of functions $f_{j} \in \mathcal{F}$,

(3) a sequence of positive numbers $\rho_{j} \rightarrow 0$,

such that $g_{j}(\zeta)=\rho_{j}^{-k} f_{j}\left(z_{j}+\rho_{j} \zeta\right)$ converges to a non-constant meromorphic function $g(\zeta)$ on $\mathbb{C}$. The zeros of $g(\zeta)$ are of multiplicity at least $d$ and has poles with multiplicity at least 2. Moreover, $g(\zeta)$ is of order at most 2.

We see that

$$
\begin{aligned}
f_{j}^{(k)}\left(z_{j}+\rho_{j} \zeta\right) & -a\left(z_{j}+\rho_{j} \zeta\right) f_{j}^{n}\left(z_{j}+\rho_{j} \zeta\right)-\psi\left(z_{j}+\rho_{j} \zeta\right) \\
& =g_{j}^{(k)}(\zeta)-\rho_{j}^{n k} a\left(z_{j}+\rho_{j} \zeta\right) g_{j}(\zeta)-\psi\left(z_{j}+\rho_{j} \zeta\right) \\
& \rightarrow g^{(k)}(\zeta)-\psi(0) .
\end{aligned}
$$


By Hurwitz's Theorem, we see that $g^{(k)}(\zeta)-\psi(0)$ has at least $m$ zeros. Now, by Milloux's inequality and Nevanlinna's First Fundamental Theorem we get

$$
\begin{aligned}
T(r, g) & \leq \bar{N}(r, g)+N\left(r, \frac{1}{g}\right)+\bar{N}\left(r, \frac{1}{g^{(k)}-\psi(0)}\right)-N\left(r, \frac{1}{g^{(k+1)}}\right)+S(r, g) \\
& \leq \frac{1}{2} N(r, g)+(k+1) \bar{N}\left(r, \frac{1}{g}\right)+\bar{N}\left(r, \frac{1}{g^{(k)}-\psi(0)}\right)+S(r, f) \\
& \leq \frac{1}{2} N(r, g)+\frac{k+1}{d} N\left(r, \frac{1}{g}\right)+\frac{1}{m} N\left(r, \frac{1}{g^{(k)}-\psi(0)}\right)+S(r, g) \\
& \leq\left(\frac{1}{2}+\frac{k+1}{d}\right) T(r, g)+\frac{1}{m}(T(r, g)+k \bar{N}(r, g))+S(r, g) \\
& \leq\left(\frac{1}{2}+\frac{k+1}{d}+\frac{1}{m}+\frac{k}{2 m}\right) T(r, g)+S(r, g) .
\end{aligned}
$$

Combining with assumption $\frac{k+1}{d}+\frac{k+2}{2 m}<\frac{1}{2}$, we get $g$ is constant. This is a contradiction. Hence $\mathcal{F}$ is a normal family, and this completes the proof of Theorem 1.1 .

We can prove Theorem 1.2 by the method of Theorem 1.1 . Since $\mathcal{F}$ is a family of holomorphic functions, so by Remark 3.2. the limit function $g$ is a non-constant entire function with zeros of multiplicity at least $d$ and $g^{(k)}(\zeta)-\psi(0)$ has at least $m$ zeros. Now, by Lemma 3.3 and Nevanlinna's First Fundamental Theorem we get

$$
\begin{aligned}
T(r, g) & \leq \bar{N}(r, g)+N\left(r, \frac{1}{g}\right)+\bar{N}\left(r, \frac{1}{g^{(k)}-\psi(0)}\right)-N\left(r, \frac{1}{g^{(k+1)}}\right)+S(r, g) \\
& \leq(k+1) \bar{N}\left(r, \frac{1}{g}\right)+\bar{N}\left(r, \frac{1}{g^{(k)}-\psi(0)}\right)+S(r, f) \\
& \leq \frac{k+1}{d} N\left(r, \frac{1}{g}\right)+\frac{1}{m} N\left(r, \frac{1}{g^{(k)}-\psi(0)}\right)+S(r, g) \\
& \leq \frac{k+1}{d} T(r, g)+\frac{1}{m}(T(r, g)+k \bar{N}(r, g))+S(r, g) \\
& \leq\left(\frac{k+1}{d}+\frac{1}{m}\right) T(r, g)+S(r, g) .
\end{aligned}
$$

Combining with assumption $\frac{k+1}{d}+\frac{1}{m}<1$, we get $g$ is constant. This is a contradiction. Hence $\mathcal{F}$ is a normal family, and this completes the proof of the Theorem 1.2 .

Proof of Theorem 1.7. Again we assume that $D=\Delta$. Suppose that $\mathcal{F}$ is not normal in $\Delta$. Then there exists at least one point $z_{0}$ such that $\mathcal{F}$ is not normal at the point $z_{0}$ in $\Delta$. Without loss of generality we assume that $z_{0}=0$. Then by Lemma 3.1, for $\alpha=-k$ there exist

(1) a sequence of complex numbers $z_{j} \rightarrow 0,\left|z_{j}\right|<r<1$,

(2) a sequence of functions $f_{j} \in \mathcal{F}$,

(3) a sequence of positive numbers $\rho_{j} \rightarrow 0$,

such that $g_{j}(\zeta)=\rho_{j}^{-k} f_{j}\left(z_{j}+\rho_{j} \zeta\right)$ converges to a non-constant meromorphic function $g(\zeta)$ on $\mathbb{C}$. The zeros of $g(\zeta)$ are of multiplicity at least $d$ and has poles with multiplicity at 
least 2. Moreover, $g(\zeta)$ is of order at most 2.

We see that

$$
\begin{aligned}
f_{j}\left(z_{j}+\rho_{j} \zeta\right) & +a\left(z_{j}+\rho_{j} \zeta\right)\left(f_{j}^{(k)}\right)^{n}\left(z_{j}+\rho_{j} \zeta\right)-\psi\left(z_{j}+\rho_{j} \zeta\right) \\
& =\rho_{j}^{k} g_{j}(\zeta)+a\left(z_{j}+\rho_{j} \zeta\right)\left(g_{j}^{(k)}\right)^{n}(\zeta)-\psi\left(z_{j}+\rho_{j} \zeta\right) \\
& \rightarrow a(0)\left(g^{(k)}\right)^{n}(\zeta)-\psi(0) .
\end{aligned}
$$

Let $a(0)=a$. By Hurwitz's Theorem, we see that $a\left(g^{(k)}\right)^{n}(\zeta)-\psi(0)$ has at least $m$ zeros. Now we invoke Lemma 3.4 for the differential polynomial $P=a\left(g^{(k)}\right)^{n}(\zeta)$ and $q=1$. Note that, $d(P)=n(\geq 2)$ and $\theta(P)=n k$. By Lemma 3.4 and Nevanlinna's First Fundamental Theorem,

$$
\begin{aligned}
T(r, g) & \leq \frac{n k+1}{n-1} \bar{N}\left(r, \frac{1}{g}\right)+\frac{1}{n-1} \bar{N}\left(r, \frac{1}{a\left(g^{(k)}\right)^{n}-\psi(0)}\right)+S(r, g) \\
& \leq \frac{n k+1}{(n-1) d} N\left(r, \frac{1}{g}\right)+\frac{1}{(n-1) m} N\left(r, \frac{1}{a\left(g^{(k)}\right)^{n}-\psi(0)}\right)+S(r, g) \\
& \leq \frac{n k+1}{(n-1) d} N\left(r, \frac{1}{g}\right)+\frac{1}{(n-1) m} T\left(r,\left(g^{(k)}\right)^{n}\right)+S(r, g) \\
& =\frac{n k+1}{(n-1) d} N\left(r, \frac{1}{g}\right)+\frac{n}{(n-1) m} T\left(r, g^{(k)}\right)+S(r, g) \\
& =\frac{n k+1}{(n-1) d} N\left(r, \frac{1}{g}\right)+\frac{n}{(n-1) m}\left(m\left(r, g^{(k)}\right)+N\left(r, g^{(k)}\right)\right)+S(r, g) \\
& \leq \frac{n k+1}{(n-1) d} T(r, g)+\frac{n}{(n-1) m}\left(m\left(r, \frac{g^{(k)}}{g}\right)+m(r, g)\right) \\
& \leq \frac{n k+1}{(n-1) d} T(r, g)+\frac{n}{(n-1) m} T(r, g)+\frac{n k}{(n-1) m} T(r, g)+S(r, g) \\
& \leq\left(\frac{n k+1}{(n-1) d}+\frac{n(1+k)}{(n-1) m}\right) T(r, g)+S(r, g) .
\end{aligned}
$$

Combining this with assumption $\frac{n k+1}{(n-1) d}+\frac{n(1+k)}{(n-1) m}<1$, we get that $g$ is a constant. This is a contradiction. Hence $\mathcal{F}$ is a normal family and this completes the proof of Theorem 1.7.

Again, we can prove Theorem 1.8 by the method of Theorem 1.7. Since $\mathcal{F}$ is a family of holomorphic functions so by Remark 3.2, the limit function $g$ is a non-constant entire function with zeros of multiplicity at least $d$ and $a\left(g^{(k)}\right)^{n}(\zeta)-\psi(0)$ has at least $m$ zeros. Now, we apply Lemma 3.4 to the differential polynomial $P=a\left(g^{(k)}\right)^{n}(\zeta)$ and $q=1$. Note that, $d(P)=n(\geq 2)$ and $\theta(P)=n k$. By Lemma 3.4 and Nevanlinna's First Fundamental 
Theorem,

$$
\begin{aligned}
T(r, g) & \leq \frac{n k+1}{n} \bar{N}\left(r, \frac{1}{g}\right)+\frac{1}{n} \bar{N}\left(r, \frac{1}{a\left(g^{(k)}\right)^{n}-\psi(0)}\right)+S(r, g) \\
& \leq \frac{n k+1}{n d} N\left(r, \frac{1}{g}\right)+\frac{1}{n m} N\left(r, \frac{1}{a\left(g^{(k)}\right)^{n}-\psi(0)}\right)+S(r, g) \\
& \leq \frac{n k+1}{n d} N\left(r, \frac{1}{g}\right)+\frac{1}{n m} T\left(r,\left(g^{(k)}\right)^{n}\right)+S(r, g) \\
& =\frac{n k+1}{n d} N\left(r, \frac{1}{g}\right)+\frac{n}{n m} T\left(r, g^{(k)}\right)+S(r, g) \\
& =\frac{n k+1}{n d} N\left(r, \frac{1}{g}\right)+\frac{1}{m}\left(m\left(r, g^{(k)}\right)+N\left(r, g^{(k)}\right)\right)+S(r, g) \\
& \leq \frac{n k+1}{n d} T(r, g)+\frac{1}{m}\left(m\left(r, \frac{g^{(k)}}{g}\right)+m(r, g)\right)+S(r, g) \\
& \leq \frac{n k+1}{n d} T(r, g)+\frac{1}{m} T(r, g)+S(r, g) \\
& \leq\left(\frac{n k+1}{n d}+\frac{1}{m}\right) T(r, g)+S(r, g) .
\end{aligned}
$$

Combining this with assumption $\frac{n k+1}{n d}+\frac{1}{m}<1$, we get $g$ is constant. This is a contradiction. Hence $\mathcal{F}$ is a normal family. This completes the proof of the Theorem 1.8 ,

Acknowledgement: The second author is thankful to the faculty and the administrative unit of School of Mathematics, Harish-Chandra Research Institute, Allahabad for their warm hospitality during the preparation of this paper.

\section{REFERENCES}

[1] L. V. Ahlfors, Complex Analysis, Third edition, McGraw-Hill, 1979.

[2] G. Datt and S. Kumar, Normality and Sharing functions, Ind. J. Pure Appl. Math., 46 (6), (2015), 853-864, DOI: 10.1007/s13226-015-0155-2.

[3] B. Deng, H. L. Qiu, D. Liu and M. L. Fang, Hayman's question on normal families concerning zero number, Comp. Var. Ellip. Equa., 59, no. 5 (2014), 616-630.

[4] G. Dethloff, T. V. Tan, N. V. Thin, Normal criteria for families of meromorphic functions, J. Math. Anal. Appl., 411 (2014), 675-683.

[5] D. Drasin, Normal families and Nevanlinna theory, Acta Math., 122 (1969), 231-263.

[6] M. L. Fang and J. M. Chang, Normal families and multiple values, Arch. Math. (Basel), 88 (2007), no. 6, 560-568.

[7] M. L. Fang and L. Zalcman, On the value distribution of $f+a\left(f^{\prime}\right)^{n}$, Sci. China Ser. A: Math., 51 (2008), no. 7, 1196-1202.

[8] W. K. Hayman, Picard values of meromorphic functions and their derivatives, Ann. Math., 70, no.2, (1959), 9-42.

[9] W. K. Hayman, Meromorphic Functions, Clarendon Press, Oxford, 1964.

[10] J. D. Hinchliffe, On a result of Chuang related to Hayman's alternative, Comput. Methods Funct. Theory, 2 (2002), 293-297.

[11] C. L. Lei, M. L. Fang and C. Zeng, Some normality criteria of meromorphic functions, Acta Math. Sci., 33B, no. 6, (2013), 1667-1674. 
[12] J. Schiff, Normal Families, Springer-Verlag, Berlin, 1993.

[13] W. Schwick, Normality criteria for families of meromophic functions, J. Anal. Math., 52 (1989), 241-289.

[14] J. Y. Xia and Y. Xu, Normal families of meromorphic functions with multiple values, J. Math. Anal. Appl., 354, (2009), 387-393.

[15] B. Xiao, W. Xiong and W. Yuan, Some normality criteria for families of meromorphic functions, Taiwanese J. Math., 19(3), (2013), 725-736. DOI: 10.11650/tjm.19.2015.4549.

[16] Y. Xu, F. Wu and L. Liao, Picard values and normal families of meromorphic functions Proc. Roy. Soc. Edinburgh, 139A, (2009), 1091-1099.

[17] C. C. Yang and H. X. Yi, Uniqueness theory of meromorphic functions, Science Press/ Kluwer Academic Publishers, 2003.

[18] L. Yang, Value Distribution Theory, Springer-Verlag, Berlin, 1993.

[19] L. Zalcman, A heuristic principle in complex function theory, Amer. Math. Monthly, 82 (1975), $813-817$.

[20] L. Zalcman, Normal families: new perspectives, Bull. Amer. Math. Soc., 35, no. 3 (1998), 215-230.

[21] S. Zeng and I. Lahiri, A normality criterion for meromorphic functions, Kodai Math. J., 35 (2012), $105-114$.

[22] S. Zeng and I. Lahiri, A normality criterion for meromorphic functions having multiple zeros, Ann. Polon. Math., 110.3 (2014), 283-294.

[23] L. Zhao and X. Wu, Normal families of holomorphic functions and multiple zeros, Bull. Belg. Math. Soc. Simon Stevin, 19 (2012), 535-547.

Department of Mathematics, Deen Dayal Upadhyaya College, University of Delhi, DELHI-110 078, INDIA

E-mail address: sanjpant@gmail.com

Department of Mathematics, University of Delhi, Delhi-110 007, India

E-mail address: pnmrani753@gmail.com 